\title{
Edge-based nonlinear diffusion for finite element approximations of convection-diffusion equations and its relation to algebraic flux-correction schemes
}

\author{
Gabriel R. Barrenechea ${ }^{1}$. Erik Burman ${ }^{2}$. \\ Fotini Karakatsani ${ }^{3}$
}

Received: 23 September 2015 / Revised: 7 March 2016 / Published online: 7 May 2016 (C) The Author(s) 2016. This article is published with open access at Springerlink.com

\begin{abstract}
For the case of approximation of convection-diffusion equations using piecewise affine continuous finite elements a new edge-based nonlinear diffusion operator is proposed that makes the scheme satisfy a discrete maximum principle. The diffusion operator is shown to be Lipschitz continuous and linearity preserving. Using these properties we provide a full stability and error analysis, which, in the diffusion dominated regime, shows existence, uniqueness and optimal convergence. Then the algebraic flux correction method is recalled and we show that the present method can be interpreted as an algebraic flux correction method for a particular definition of the flux limiters. The performance of the method is illustrated on some numerical test cases in two space dimensions.
\end{abstract}

Mathematics Subject Classification 65 N30 $\cdot 65$ N12

\section{Introduction}

For an open bounded polygonal (polyhedral) domain $\Omega \subseteq \mathbb{R}^{d}, d=2,3$, with Lipschitz boundary, we consider in this work the steady-state convection-diffusionreaction equation

Gabriel R. Barrenechea

gabriel.barrenechea@strath.ac.uk

1 Department of Mathematics and Statistics, University of Strathclyde, 26 Richmond Street, Glasgow G1 1XH, UK

2 Department of Mathematics, University College London, Gower Street, London WC1E 6BY, UK

3 Department of Mathematics, University of Chester, Thornton Science Park,

Chester $\mathrm{CH} 2$ 4NU, UK 


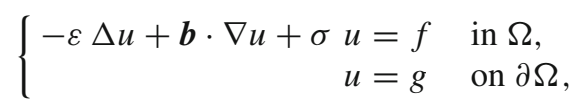

where $\varepsilon>0$ is the diffusion coefficient, $\boldsymbol{b} \in L^{\infty}(\Omega)^{2}$ is a solenoidal convective field, $\sigma>0$ is a real constant, and $f \in L^{2}(\Omega), g \in H^{\frac{1}{2}}(\partial \Omega)$, are given data. In this work we adopt the standard notation for Sobolev spaces. In particular, for $D \subset \mathbb{R}^{d}$ we denote $(\cdot, \cdot)_{D}$ the $L^{2}(D)$ [or $L^{2}(D)^{d}$ ] inner product, and by $\|\cdot\|_{l, D}\left(|\cdot|_{l, D}\right)$ the norm (seminorm) in $H^{l}(D)$ [with the usual convention that $H^{0}(D)=L^{2}(D)$ ].

The weak form of problem (1.1) is: Find $u \in H^{1}(\Omega)$ such that $u=g$ on $\partial \Omega$ and

$$
a(u, v)=(f, v)_{\Omega} \quad \forall v \in H_{0}^{1}(\Omega),
$$

where the bilinear form $a$ is given by

$$
a(u, v):=\varepsilon(\nabla u, \nabla v)_{\Omega}+(\boldsymbol{b} \cdot \nabla u, v)_{\Omega}+\sigma(u, v)_{\Omega} .
$$

The weak problem (1.2) has a unique solution $u \in H^{1}(\Omega)$ and its solution satisfies the following maximum principle (see [10]).

Definition 1 (Maximum principle) Assume that $f \geq 0, g \geq 0$ (resp. $\leq 0$ ) and the solution $u$ of (1.2) is smooth enough. Then, if $\sigma=0$ and $u$ attains a strict minimum (resp. maximum) at an interior point $\tilde{x} \in \Omega$, then $u$ is constant in $\Omega$. If $\sigma>0$, then the same conclusion remains valid if we suppose in addition that $u(\tilde{x})<0$ [resp. $u(\tilde{x})>0]$.

This work deals with the development of a method that satisfies the discrete analogue of the last definition. The quest for such a method has been a constant for the last couple of decades. Several methods have been proposed over the years, both in the finite element and finite volume contexts (see [21] for a review). Overall, the common point of all discretisations that satisfy a discrete maximum principle (DMP) is that they add some diffusion to the equations. This extra diffusion can lead to a linear method, but it is a well-known fact that such a method will provide very diffused numerical solutions, which will converge suboptimally. Due to the previous fact, several methods that add nonlinear diffusion have been proposed.

One approach has been to add a so-called shock-capturing term to the finite element formulation. This typically amounts to a nonlinear diffusion term where the diffusion coefficient depends nonlinearly on the finite element residual, making it large in the zones where the solution is underresolved, but vanish in smooth regions. An analysis showing that nonlinear shock capturing methods may lead to a DMP was first proposed in [5], and then developed further for the Laplace operator in [6], and for the convection-diffusion equation in [7]. For a review of shock capturing methods, designed to reduce spurious oscillations, without necessarily satisfying a DMP, see [14]. More recent nonlinear discretisations, these ones based on the idea of blending in order to satisfy the DMP, are the works [1,9], where the emphasis has been given to prove the convergence to an entropy solution. Most shock capturing techniques suffer from the strong nonlinearity introduced when the diffusion coefficient is made to depend on the finite element residual (and therefore the gradient of the approximation 
function). Because of this the analysis of such methods is incomplete even when linear model problems with constant coefficients are considered. In particular, in most cases uniqueness of solutions can not be proved, and the convergence theory is incomplete.

On the other hand, driven initially by the design of explicit time stepping schemes for compressible flows, so called flux corrected transport (FCT) schemes and the related algebraic flux correction (AFC) schemes were introduced [15, 19,20]. These schemes act on the algebraic level by first modifying the system matrix so that it has suitable properties to make the system monotonous, while perturbing the method as little as possible. In the most elementary case the system matrix is simply perturbed to make it an M-matrix, resulting in a linear method. This crude strategy, however, necessarily results in a first order scheme. Then, AFC schemes introduce a nonlinear switch, or flux limiter, thus making the low order monotone scheme active only in the zones where the DMP may be violated. These schemes have also resisted mathematical analysis for a long time, but a number of results have been proved recently in [2,3]. Indeed, in these references, existence of solutions and positivity have been proved, and a first error analysis has been performed. Nevertheless, it was shown that the DMP, and even the convergence of the discrete solution to the continuous one, depend on the geometry of the mesh.

Another approach to combine monotone (low order) finite element methods with linear diffusion and high order FEM using flux-limiters was proposed very recently in [13]. It then appears that a cross pollination between the idea of AFC and shockcapturing could be fruitful.

The objective of the present paper is to further bridge the gap between the shock capturing approach and the algebraic flux correction. Indeed we will consider a generalisation of the shock-capturing term first introduced in [4] to several dimensions, using an anisotropic diffusion operator along element edges similar to that introduced in [7]. We show that the resulting scheme satisfies the DMP and give an analysis of the method. In particular we show that the new shock capturing term is Lipschitz continuous, and, if the mesh is sufficiently regular, linearity preserving (see Sect. 2.1), which allows us to improve greatly on previous results. In Sect. 2.2 we prove existence of solutions, the discrete maximum principle, and noticeably, uniqueness in the diffusion dominated regime. We then show error estimates, which, thanks to the combined use of linearity preservation and Lipschitz continuity, turn out to be optimal in the diffusion dominated regime, for a special class of meshes (see Sect. 3). In Sect. 4, we revisit the design principles of AFC and show that the proposed shock-capturing term can be interpreted as an AFC scheme using a special flux, allowing both for a DMP and Lipschitz continuity. Some numerical results are finally shown in Sect. 5.

\subsection{Notations}

We now introduce some notation that will be needed for the discrete setting. We consider a family $\left\{\mathscr{T}_{h}\right\}_{h>0}$ of shape-regular triangulations of $\Omega$ consisting of disjoint $d$-simplices $K$. We define $h_{K}:=\operatorname{diam}(K)$, and $h=\max \left\{h_{K}: K \in \mathscr{T}_{h}\right\}$. We associate with the triangulation $\mathscr{T}_{h}$ the finite element spaces 


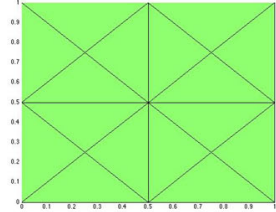

(a)

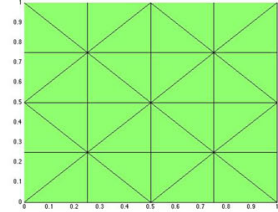

(b)

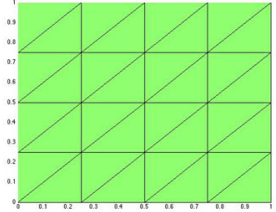

(c)

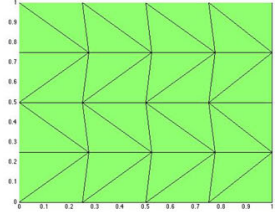

(d)

Fig. 1 In two dimensions, meshes (a-c) are examples of symmetric meshes. Mesh (d) is a non-symmetric, non-Delaunay mesh

$$
\mathcal{V}_{h}:=\left\{\chi \in H^{1}(\Omega):\left.\chi\right|_{K} \in \mathbb{P}_{1}(K) \forall K \in \mathscr{T}_{h}\right\}, \quad \text { and } \quad \mathcal{V}_{h}^{0}:=\mathcal{V}_{h} \cap H_{0}^{1}(\Omega),
$$

where $\mathbb{P}_{\ell}(D)$ is the space of polynomials of degree at most $\ell$ on $D$. The nodes of $\mathscr{T}_{h}$ are denoted by $\left\{x_{i}\right\}_{i=1}^{N}$, and the usual associated basis functions of $\mathcal{V}_{h}$ are denoted by $\left\{\psi_{i}\right\}_{i=1}^{N}$.

We let $\mathscr{E}_{h}$ be the set of the interior edges of $\mathscr{T}_{h}$. For every edge $E \in \mathscr{E}_{h}$, we define $h_{E}:=|E|$ and $\omega_{E}:=\left\{K \in \mathscr{T}_{h}: K \cap E \neq \emptyset\right\}$, and fix one unit tangent vector, denoted by $\boldsymbol{t}$.

For an interior node $x_{i}$, we define the associated edges $\mathscr{E}_{i}:=\left\{E \in \mathscr{E}_{h}: x_{i} \in E\right\}$ and the subset of $\mathbb{R}^{d}$ defined by the union of all elements $K$ sharing the node $x_{i}$, $\Omega_{i}:=\left\{x \in \bar{\Omega}: \exists K \in \mathscr{T}_{h}: x \in K\right.$ and $\left.x_{i} \in K\right\}$, and the set

$$
S_{i}:=\left\{j \in\{1, \ldots, N\} \backslash\{i\}: x_{j} \text { shares an internal edge with } x_{i}\right\} .
$$

Finally, we will say that the triangulation $\mathscr{T}_{h}$ is symmetric with respect to its internal nodes if for every internal node $x_{i}$ the following holds: for all $j \in S_{i}$ there exists $k \in S_{i}$ such that $x_{j}-x_{i}=-\left(x_{k}-x_{i}\right)$ (see Fig. 1 for examples in two space dimensions).

\section{The nonlinear discretisation}

The standard finite element method for the problem (1.2) takes the form: find $u_{h} \in \mathcal{V}_{h}$ such that $u_{h}-u_{b h} \in \mathcal{V}_{h}^{0}$ and

$$
a\left(u_{h}, v_{h}\right)=\left(f, v_{h}\right)_{\Omega} \quad \forall v_{h} \in \mathcal{V}_{h}^{0}
$$

Here, $u_{b h} \in \mathcal{V}_{h}$ is introduced to approximate the boundary condition $g$. Then, we propose the following stabilised method to discretise (1.2): find $u_{h} \in \mathcal{V}_{h}$ such that $u_{h}-u_{b h} \in \mathcal{V}_{h}^{0}$ and

$$
a_{h}\left(u_{h} ; v_{h}\right):=a\left(u_{h}, v_{h}\right)+d_{h}\left(u_{h} ; u_{h}, v_{h}\right)=\left(f, v_{h}\right)_{\Omega} \quad \forall v_{h} \in \mathcal{V}_{h}^{0}
$$

The stabilisation term $d_{h}(\cdot ; \cdot, \cdot)$ is defined by

$$
d_{h}\left(w_{h} ; u_{h}, v_{h}\right)=\sum_{E \in \mathscr{E}_{h}} \gamma_{0} h_{E}^{d} \alpha_{E}\left(w_{h}\right)\left(\partial_{t} u_{h}, \partial_{t} v_{h}\right)_{E} .
$$


Here, $\gamma_{0}>0$, and $\alpha_{E}: \mathcal{V}_{h} \rightarrow[0,1]$ is defined as follows. First, for $w_{h} \in \mathcal{V}_{h}$, we define $\xi_{w_{h}}$ as the unique element in $\mathcal{V}_{h}^{0}$ whose nodal values are given by

$$
\xi_{w_{h}}\left(x_{i}\right):= \begin{cases}\frac{\left|\sum_{j \in S_{i}} w_{h}\left(x_{i}\right)-w_{h}\left(x_{j}\right)\right|}{\sum_{j \in S_{i}}\left|w_{h}\left(x_{i}\right)-w_{h}\left(x_{j}\right)\right|}, & \text { if } \sum_{j \in S_{i}}\left|w_{h}\left(x_{i}\right)-w_{h}\left(x_{j}\right)\right| \neq 0 \\ 0, & \text { otherwise. }\end{cases}
$$

Then, on each $E, \alpha_{E}$ is defined by

$$
\alpha_{E}\left(w_{h}\right):=\max _{x \in E}\left[\xi_{w_{h}}(x)\right]^{p}, \quad p \in[1,+\infty) .
$$

The value for $p$ will determine the rate of decay of the numerical diffusion with the distance to the critical points. A value closer to 1 will add more diffusion in the far field, while a larger value will make the diffusion vanish faster, but on the other hand, increasing $p$ may make the nonlinear system more difficult to solve. In principle, as $p$ goes to infinity the method will add the perturbations only in points with local extrema. In our calculations we have tested several different values for $p$, and have presented those for $p=1,4,8$, and 10 . The higher values provide better numerical results, while keeping the nonlinear solver converging within a reasonable number of iterations. In Sect. 5 below we present a more detailed study of the behavior of the nonlinear solver with respect to the value of $p$. We finally stress the fact that, for any value of $p$, the function $\alpha_{E}\left(w_{h}\right)$ is equal to 1 if $w_{h}$ has a local extremum in one of the end points of the edge $E$. This property is of fundamental importance for the proof of the discrete maximum principle below.

\subsection{Properties of $d_{h}(\cdot ; \cdot, \cdot)$}

We start noticing that

$$
\sum_{j \in S_{i}}\left|w_{h}\left(x_{i}\right)-w_{h}\left(x_{j}\right)\right|=\left.0 \Longrightarrow w_{h}\right|_{\Omega_{i}}=c \in \mathbb{R}
$$

This prevents the method from adding artificial diffusion to the equations in regions in which the solution is constant. Moreover, the method is as well linearity preserving if the mesh is symmetric with respect to its interior nodes. In fact, if $E \in \mathscr{E}_{h}$ has endpoints $x_{i}$ and $x_{j}$, and $v_{h} \in \mathbb{P}_{1}\left(\omega_{E}\right)$, then

$$
\sum_{l \in S_{i}} v_{h}\left(x_{i}\right)-v_{h}\left(x_{l}\right)=0 \text { and } \sum_{l \in S_{j}} v_{h}\left(x_{j}\right)-v_{h}\left(x_{l}\right)=0
$$

which gives $\alpha_{E}\left(v_{h}\right)=0$. Then, the method does not add extra diffusion in smooth regions, whenever the mesh is sufficiently structured. We now state this in a more precise way. Let us decompose the stabilisation term $d_{h}$ as the sum of edge contributions as follows: 


$$
\begin{aligned}
d_{h}\left(u_{h} ; v_{h}, z_{h}\right) & =\sum_{E \in \mathscr{E}_{h}} d_{E}\left(u_{h} ; v_{h}, z_{h}\right) \\
\text { with } d_{E}\left(u_{h} ; v_{h}, z_{h}\right) & :=\gamma_{0} h_{E}^{d} \alpha_{E}\left(u_{h}\right)\left(\partial_{t} v_{h}, \partial_{t} z_{h}\right)_{E} .
\end{aligned}
$$

Then, if the mesh is symmetric with respect to its internal nodes and $E \in \mathscr{E} h$, whenever $v_{h} \in \mathbb{P}_{1}\left(\omega_{E}\right)$, the edge diffusion vanishes, this is

$$
d_{E}\left(v_{h} ; w_{h}, z_{h}\right)=0 \quad \forall w_{h}, z_{h} \in \mathcal{V}_{h} .
$$

As a consequence, if, for a given node $x_{i}$, with associated basis function $\psi_{i}$, we denote the extended macro element $\tilde{\Omega}_{i}:=\cup_{E \in \mathcal{E}_{i}} \omega_{E}$, then

$$
d_{h}\left(v_{h} ; w_{h}, \psi_{i}\right)=0, \quad \forall w_{h} \in \mathcal{V}_{h} \text { and } \forall v_{h}:\left.v_{h}\right|_{\tilde{\Omega}_{i}} \in P_{1}\left(\tilde{\Omega}_{i}\right)
$$

The next step is to show that $d_{h}(\cdot ; \cdot, \cdot)$ is continuous. More precisely, it is Lipschitz continuous, and the next result is the first step towards this.

Lemma 1 For any $v_{h}, w_{h} \in \mathcal{V}_{h}$, and any given internal node $x_{i}$, the following holds

$$
\left|\xi_{v_{h}}\left(x_{i}\right)-\xi_{w_{h}}\left(x_{i}\right)\right| \leq 4 \frac{\sum_{E \in \mathscr{E}_{i}} h_{E}\left|\partial_{t}\left(v_{h}-w_{h}\right)\right|}{\sum_{E \in \mathscr{E}_{i}} h_{E}\left(\left|\partial_{t} v_{h}\right|+\left|\partial_{t} w_{h}\right|\right)}
$$

Proof It is enough to suppose that $\sum_{j \in S_{i}}\left|v_{h}\left(x_{i}\right)-v_{h}\left(x_{j}\right)\right|>0$ and $\sum_{j \in S_{i}} \mid w_{h}\left(x_{i}\right)-$ $w_{h}\left(x_{j}\right) \mid>0$, otherwise the claim is obvious. A quick calculation gives

$$
\begin{aligned}
\left|\xi_{v_{h}}\left(x_{i}\right)-\xi_{w_{h}}\left(x_{i}\right)\right| & || \frac{\sum_{j \in S_{i}} v_{h}\left(x_{i}\right)-v_{h}\left(x_{j}\right) \mid}{\sum_{E \in \mathscr{E}_{i}} h_{E}\left|\partial_{\boldsymbol{t}} v_{h}\right|}-\frac{\left|\sum_{j \in S_{i}} w_{h}\left(x_{i}\right)-w_{h}\left(x_{j}\right)\right|}{\sum_{E \in \mathscr{E}_{i}} h_{E}\left|\partial_{\boldsymbol{t}} w_{h}\right|} \mid \\
\leq & \left|\frac{\left|\sum_{j \in S_{i}} v_{h}\left(x_{i}\right)-v_{h}\left(x_{j}\right)\right|-\left|\sum_{j \in S_{i}} w_{h}\left(x_{i}\right)-w_{h}\left(x_{j}\right)\right|}{\sum_{E \in \mathscr{E}_{i}} h_{E}\left|\partial_{\boldsymbol{t}} v_{h}\right|}\right| \\
& +\left|\sum_{j \in S_{i}} w_{h}\left(x_{i}\right)-w_{h}\left(x_{j}\right)\right|\left|\frac{1}{\sum_{E \in \mathscr{E}_{i}} h_{E}\left|\partial_{\boldsymbol{t}} v_{h}\right|}-\frac{1}{\sum_{E \in \mathscr{E}_{i}} h_{E}\left|\partial_{\boldsymbol{t}} w_{h}\right|}\right| \\
\leq & \frac{\sum_{E \in \mathscr{E}_{i}} h_{E}\left|\partial_{\boldsymbol{t}}\left(v_{h}-w_{h}\right)\right|}{\sum_{E \in \mathscr{E}_{i}} h_{E}\left|\partial_{\boldsymbol{t}} v_{h}\right|} \\
& +\frac{\left|\sum_{j \in S_{i}} w_{h}\left(x_{i}\right)-w_{h}\left(x_{j}\right)\right|\left|\sum_{E \in \mathscr{E}_{i}} h_{E}\left(\left|\partial_{\boldsymbol{t}} w_{h}\right|-\left|\partial_{\boldsymbol{t}} v_{h}\right|\right)\right|}{\sum_{E \in \mathscr{E}_{i}} h_{E}\left|\partial_{\boldsymbol{t}} v_{h}\right| \sum_{E \in \mathscr{E}_{i}} h_{E}\left|\partial_{\boldsymbol{t}} w_{h}\right|} \\
\leq & 2 \frac{\sum_{E \in \mathscr{E}_{i}} h_{E}\left|\partial_{\boldsymbol{t}}\left(v_{h}-w_{h}\right)\right|}{\sum_{E \in \mathscr{E}_{i}} h_{E}\left|\partial_{\boldsymbol{t}} v_{h}\right|} .
\end{aligned}
$$


The following estimate can be proved in an analogous way

$$
\left|\xi_{v_{h}}\left(x_{i}\right)-\xi_{w_{h}}\left(x_{i}\right)\right| \leq 2 \frac{\sum_{E \in \mathscr{E}_{i}} h_{E}\left|\partial_{t}\left(v_{h}-w_{h}\right)\right|}{\sum_{E \in \mathscr{E}_{i}} h_{E}\left|\partial_{t} w_{h}\right|}
$$

Then,

$$
\begin{aligned}
& \left|\xi_{v_{h}}\left(x_{i}\right)-\xi_{w_{h}}\left(x_{i}\right)\right| \\
& \quad \leq 2 \min \left\{\frac{1}{\sum_{E \in \mathscr{E}_{i}} h_{E}\left|\partial_{\boldsymbol{t}} v_{h}\right|}, \frac{1}{\sum_{E \in \mathscr{E}_{i}} h_{E}\left|\partial_{\boldsymbol{t}} w_{h}\right|}\right\} \sum_{E \in \mathscr{E}_{i}} h_{E}\left|\partial_{t}\left(v_{h}-w_{h}\right)\right|,
\end{aligned}
$$

which gives the desired result upon applying the estimate $\min \left\{a^{-1}, b^{-1}\right\} \leq \frac{2}{a+b}$, for two positive numbers $a$ and $b$.

The Lipschitz continuity of $d_{h}(\cdot ; \cdot, \cdot)$ appears then as a consequence of the previous result.

Lemma 2 The nonlinear form $d_{h}(\cdot ; \cdot, \cdot)$ is Lipschitz continuous. More precisely, there exists $C_{\text {lip }}>0$, independent of $h$, such that, for all $v_{h}, w_{h}, z_{h} \in \mathcal{V}_{h}$, the following holds

$$
\left|d_{h}\left(v_{h} ; v_{h}, z_{h}\right)-d_{h}\left(w_{h} ; w_{h}, z_{h}\right)\right| \leq C_{\text {lip }} \gamma_{0} h\left|v_{h}-w_{h}\right|_{1, \Omega}\left|z_{h}\right|_{1, \Omega}
$$

Proof We have

$$
\begin{aligned}
& d_{h}\left(v_{h} ; v_{h}, z_{h}\right)-d_{h}\left(w_{h} ; w_{h}, z_{h}\right) \\
& =\sum_{E \in \mathscr{E}_{h}} \gamma_{0} h_{E}^{d}\left(\alpha_{E}\left(v_{h}\right) \partial_{t} v_{h}-\alpha_{E}\left(w_{h}\right) \partial_{t} w_{h}, \partial_{t} z_{h}\right)_{E} \\
& =\sum_{E \in \mathscr{E}_{h}} \gamma_{0} h_{E}^{d} \alpha_{E}\left(v_{h}\right)\left(\partial_{t} v_{h}-\partial_{t} w_{h}, \partial_{t} z_{h}\right)_{E} \\
& \quad+\gamma_{0} h_{E}^{d}\left(\alpha_{E}\left(v_{h}\right)-\alpha_{E}\left(w_{h}\right)\right)\left(\partial_{t} w_{h}, \partial_{t} z_{h}\right)_{E} .
\end{aligned}
$$

The first term in the above estimate is bounded using the fact that $\left|\alpha_{E}\left(v_{h}\right)\right| \leq 1$, the Cauchy-Schwarz inequality, a local trace inequality, and the shape regularity of the mesh sequence, to give

$$
\sum_{E \in \mathscr{E}_{h}} \gamma_{0} h_{E}^{d} \alpha_{E}\left(v_{h}\right)\left(\partial_{t} v_{h}-\partial_{t} w_{h}, \partial_{t} z_{h}\right)_{E} \leq C \gamma_{0} h\left|v_{h}-w_{h}\right|_{1, \Omega}\left|z_{h}\right|_{1, \Omega}
$$

The second term is bounded next. For this, a general edge $E \in \mathscr{E}_{h}$ will be considered as having $x_{i}$ and $x_{j}$ as endpoints, where $x_{i}$ is chosen to be the vertex such that $\alpha_{E}\left(v_{h}\right)=$ $\xi_{v_{h}}^{p}\left(x_{i}\right)$. We then divide $\mathscr{E}_{h}=E_{1} \cup E_{2}$, where

$$
E_{1}:=\left\{E \in \mathscr{E}_{h}: \alpha_{E}\left(v_{h}\right)=\xi_{v_{h}}^{p}\left(x_{i}\right), \alpha_{E}\left(w_{h}\right)=\xi_{w_{h}}^{p}\left(x_{i}\right)\right\}
$$




$$
E_{2}:=\left\{E \in \mathscr{E}_{h}: \alpha_{E}\left(v_{h}\right)=\xi_{v_{h}}^{p}\left(x_{i}\right), \alpha_{E}\left(w_{h}\right)=\xi_{w_{h}}^{p}\left(x_{j}\right)\right\}
$$

and the second term in (2.10) reduces to

$$
\begin{aligned}
& \sum_{E \in E_{1}} \gamma_{0} h_{E}^{d}\left(\left(\xi_{v_{h}}^{p}\left(x_{i}\right)-\xi_{w_{h}}^{p}\left(x_{i}\right)\right) \partial_{t} w_{h}, \partial_{t} z_{h}\right)_{E} \\
& \quad+\sum_{E \in E_{2}} \gamma_{0} h_{E}^{d}\left(\left(\xi_{v_{h}}^{p}\left(x_{i}\right)-\xi_{w_{h}}^{p}\left(x_{j}\right)\right) \partial_{t} w_{h}, \partial_{t} z_{h}\right)_{E}
\end{aligned}
$$

We now remark that for two numbers $a, b \in[0,1]$ we have

$$
\left|a^{p}-b^{p}\right|=|a-b| \sum_{l=0}^{p-1} a^{l} b^{p-1-l} \leq p|a-b|,
$$

and the term in $E_{1}$ is bounded using Lemma 1 . In fact, from the shape regularity of the mesh sequence there exists $C>0$, independent of $h$, such that for all $E, F \in \mathscr{E}_{i}, h_{F} \leq$ $C h_{E}$. Moreover, the number of edges in $\mathscr{E}_{i}$ is uniformly bounded, independently of $h$. Then, using Cauchy-Schwarz's inequality and a local trace inequality we arrive at

$$
\begin{aligned}
& \sum_{E \in E_{1}} \gamma_{0} h_{E}^{d}\left(\left(\xi_{v_{h}}^{p}\left(x_{i}\right)-\xi_{w_{h}}^{p}\left(x_{i}\right)\right) \partial_{\boldsymbol{t}} w_{h}, \partial_{\boldsymbol{t}} z_{h}\right)_{E} \\
& \leq p \sum_{E \in E_{1}} \gamma_{0} h_{E}^{d}\left(\left|\xi_{v_{h}}\left(x_{i}\right)-\xi_{w_{h}}\left(x_{i}\right)\right| \partial_{\boldsymbol{t}} w_{h}, \partial_{\boldsymbol{t}} z_{h}\right)_{E} \\
& \leq p \sum_{E \in E_{1}} \gamma_{0} h_{E}^{d}\left(4 \frac{\sum_{F \in \mathscr{E}_{i}} h_{F}\left|\partial_{\boldsymbol{t}}\left(v_{h}-w_{h}\right)\right|_{F} \mid}{\sum_{F \in \mathscr{E}_{i}} h_{F}\left(\left.\left|\partial_{\boldsymbol{t}} v_{h}\right| F|+| \partial_{\boldsymbol{t}} w_{h}\right|_{F} \mid\right)}\left|\partial_{\boldsymbol{t}} w_{h}\right|,\left|\partial_{\boldsymbol{t}} z_{h}\right|\right)_{E} \\
& \leq 4 p \gamma_{0} \sum_{E \in E_{1}} h_{E}^{d}\left(\sum_{F \in \mathscr{E}_{i}}\left|\partial_{\boldsymbol{t}}\left(v_{h}-w_{h}\right)\right|_{F}|,| \partial_{\boldsymbol{t}} z_{h} \mid\right)_{E} \\
& \leq C \gamma_{0} h\left|v_{h}-w_{h}\right| 1, \Omega\left|z_{h}\right|_{1, \Omega} .
\end{aligned}
$$

The sum over $E_{2}$ is bounded next. First, using (2.12) we get

$$
\begin{aligned}
& \sum_{E \in E_{2}} \gamma_{0} h_{E}^{d}\left(\left(\xi_{v_{h}}^{p}\left(x_{i}\right)-\xi_{w_{h}}^{p}\left(x_{j}\right)\right) \partial_{t} w_{h}, \partial_{t} z_{h}\right)_{E} \\
& =\sum_{E \in E_{2}} \gamma_{0} h_{E}^{d}\left(\left(\xi_{v_{h}}^{p}\left(x_{i}\right)-\xi_{w_{h}}^{p}\left(x_{i}\right)\right) \partial_{\boldsymbol{t}} w_{h}, \partial_{\boldsymbol{t}} z_{h}\right)_{E} \\
& \quad+\sum_{E \in E_{2}} \gamma_{0} h_{E}^{d}\left(\left(\xi_{w_{h}}^{p}\left(x_{i}\right)-\xi_{w_{h}}^{p}\left(x_{j}\right)\right) \partial_{\boldsymbol{t}} w_{h}, \partial_{\boldsymbol{t}} z_{h}\right)_{E} \\
& \leq C \gamma_{0} h\left|v_{h}-w_{h}\right|_{1, \Omega}\left|z_{h}\right|_{1, \Omega}+\sum_{E \in E_{2}} \gamma_{0} h_{E}^{d}\left(\left(\xi_{w_{h}}^{p}\left(x_{i}\right)-\xi_{w_{h}}^{p}\left(x_{j}\right)\right) \partial_{\boldsymbol{t}} w_{h}, \partial_{\boldsymbol{t}} z_{h}\right)_{E}
\end{aligned}
$$


In an analogous way we obtain

$$
\begin{aligned}
& \sum_{E \in E_{2}} \gamma_{0} h_{E}^{d}\left(\left(\xi_{v_{h}}^{p}\left(x_{i}\right)-\xi_{w_{h}}^{p}\left(x_{j}\right)\right) \partial_{\boldsymbol{t}} w_{h}, \partial_{\boldsymbol{t}} z_{h}\right)_{E} \leq C \gamma_{0} h\left|v_{h}-w_{h}\right|_{1, \Omega}\left|z_{h}\right|_{1, \Omega} \\
& \quad+\sum_{E \in E_{2}} \gamma_{0} h_{E}^{d}\left(\left(\xi_{v_{h}}^{p}\left(x_{i}\right)-\xi_{v_{h}}^{p}\left(x_{j}\right)\right) \partial_{\boldsymbol{t}} w_{h}, \partial_{\boldsymbol{t}} z_{h}\right)_{E} .
\end{aligned}
$$

Hence

$$
\begin{aligned}
& \sum_{E \in E_{2}} \gamma_{0} h_{E}^{d}\left(\left(\xi_{v_{h}}^{p}\left(x_{i}\right)-\xi_{w_{h}}^{p}\left(x_{j}\right)\right) \partial_{t} w_{h}, \partial_{t} z_{h}\right)_{E} \\
& \leq C \gamma_{0} h\left|v_{h}-w_{h}\right|_{1, \Omega}\left|z_{h}\right|_{1, \Omega} \\
& \quad+\sum_{E \in E_{2}} \gamma_{0} h_{E}^{d} \min \left\{\left(\xi_{v_{h}}^{p}\left(x_{i}\right)-\xi_{v_{h}}^{p}\left(x_{j}\right)\right)\left(\partial_{\boldsymbol{t}} w_{h}, \partial_{t} z_{h}\right)_{E},\right. \\
& \left.\quad\left(\xi_{w_{h}}^{p}\left(x_{i}\right)-\xi_{w_{h}}^{p}\left(x_{j}\right)\right)\left(\partial_{t} w_{h}, \partial_{\boldsymbol{t}} z_{h}\right)_{E}\right\} \\
& \leq C \gamma_{0} h\left|v_{h}-w_{h}\right|_{1, \Omega}\left|z_{h}\right|_{1, \Omega},
\end{aligned}
$$

since the last term in the middle inequality is always non-positive, since by construction, for $E \in E_{2}, \xi_{v_{h}}^{p}\left(x_{i}\right)-\xi_{v_{h}}^{p}\left(x_{j}\right) \geq 0$ and $\xi_{w_{h}}^{p}\left(x_{i}\right)-\xi_{w_{h}}^{p}\left(x_{j}\right) \leq 0$. The result then follows collecting (2.10)-(2.13).

Remark 1 It is worth remarking that a modification of the method can be introduced in such a way that the method becomes linearity preserving on general meshes. This modification is based on the introduction of appropriate weights in the definition of $\xi_{w_{h}}$. More precisely, instead of its original definition (2.4), we can introduce the following modified one: for $w_{h} \in \mathcal{V}_{h}$ and any internal node $x_{i}$

$$
\xi_{w_{h}}\left(x_{i}\right):= \begin{cases}\frac{\left|\sum_{j \in S_{i}} \beta_{i j}\left(w_{h}\left(x_{j}\right)-w_{h}\left(x_{i}\right)\right)\right|}{\sum_{j \in S_{i}} \beta_{i j}\left|w_{h}\left(x_{i}\right)-w_{h}\left(x_{j}\right)\right|} & \text { if } \sum_{j \in S_{i}} \beta_{i j}\left|w_{h}\left(x_{j}\right)-w_{h}\left(x_{i}\right)\right| \neq 0, \\ 0 & \text { otherwise. }\end{cases}
$$

The coefficients $\beta_{i j}$ are designed in such a way that they satisfy the linearirty preservation property. Denoting $\boldsymbol{\tau}_{i j}=x_{j}-x_{i}$, this condition reads

$\forall v \in \mathbb{P}_{1}\left(\Omega_{i}\right) \quad \sum_{j \in S_{i}} \beta_{i j}\left(v\left(x_{j}\right)-v\left(x_{i}\right)\right)=\sum_{j \in S_{i}} \beta_{i j} \nabla v \cdot \boldsymbol{\tau}_{i j}=\nabla v \cdot\left(\sum_{j \in S_{i}} \beta_{i j} \boldsymbol{\tau}_{i j}\right)=0$,

which is equivalent to imposing

$$
\sum_{j \in S_{i}} \beta_{i j} \boldsymbol{\tau}_{i j}=\mathbf{0}
$$


The Eq. (2.14) is a first restriction that the coefficients have to satisfy. A further restriction on $\beta_{i j}$ is their strict positivity. Then, we impose

$$
\beta_{i j} \geq C_{0}>0
$$

where the value of $C_{0}$ is of no great importance. Finally, in case the mesh is symetric with respect to its interior nodes, then $\beta_{i j}=1$ for all $i, j$ should be an acceptable (and preferred) solution. Then, we find $\beta_{i j}$ as the solution of the following problem: for all internal node $x_{i}$, find

$$
\left(\beta_{i j}\right)_{j \in S_{i}}=\operatorname{argmin}\left\{\sum_{j \in S_{i}}\left|\delta_{i j}-1\right|^{2}:\left\{\delta_{i j}\right\} \text { satisfies the restrictions (2.14), (2.15) }\right\} \text {. }
$$

The same results that are presented for the original definition of $\xi$ in (2.4) can be obtained for the present modification. For simplicity of the presentation, and also to avoid the computational complexity of solving the constrained optimisation problem (2.16), we have preferred to use in the rest of the paper the original definition (2.4).

\subsection{Solvability of the discrete problem}

This section is devoted to analyse the existence of solutions for (2.2). It is interesting to remark that, thanks to the Lipschitz continuity of $d_{h}(\cdot ; \cdot, \cdot)$, the solution can be proved to be unique in the diffusion-dominated regime.

Lemma 3 Let $T_{h}: \mathcal{V}_{h}^{0} \rightarrow\left[\mathcal{V}_{h}^{0}\right]^{\prime}$ be the operator defined by

$$
\left[T_{h} z_{h}, v_{h}\right]=a_{h}\left(z_{h}+u_{b h} ; v_{h}\right)-\left(f, v_{h}\right)_{\Omega}, \quad z_{h}, v_{h} \in \mathcal{V}_{h}^{0}
$$

where $[\cdot, \cdot]$ denotes the duality pairing between $\mathcal{V}_{h}^{0}$ and its dual. Then,

$$
\left[T_{h} z_{h}, z_{h}\right] \geq c_{1}\left|z_{h}\right|_{1, \Omega}^{2}-c_{2}\left(\left\|u_{b h}\right\|_{1, \Omega}^{2}+\|f\|_{0, \Omega}^{2}\right),
$$

where $c_{1}, c_{2}$ are positive constants independent of $z_{h}, f$, and $g$.

Proof For this proof only, we will consider constants $C>0$ that may depend on the physical coefficients. From the definition of $a$ it follows that

$$
a\left(z_{h}, z_{h}\right)=\varepsilon\left|z_{h}\right|_{1, \Omega}^{2}+\left(\sigma z_{h}, z_{h}\right) \geq \varepsilon\left|z_{h}\right|_{1, \Omega}^{2} .
$$

Moreover, the definition of $d_{h}(\cdot ; \cdot, \cdot)$ and the fact that $0 \leq \alpha_{E}\left(z_{h}+u_{b h}\right)$ give

$$
d_{h}\left(z_{h}+u_{b h} ; z_{h}, z_{h}\right)=\sum_{E \in \mathscr{E}_{h}} \gamma_{0} h_{E}^{d} \alpha_{E}\left(z_{h}+u_{b h}\right)\left\|\partial_{t} z_{h}\right\|_{0, E}^{2} \geq 0
$$


Then, the definition of the operator $T_{h}$ gives

$$
\left[T_{h} z_{h}, z_{h}\right] \geq \varepsilon\left|z_{h}\right|_{1, \Omega}^{2}+a\left(u_{b h}, z_{h}\right)+d_{h}\left(z_{h}+u_{b h} ; u_{b h}, z_{h}\right)-\left(f, z_{h}\right)_{\Omega} .
$$

Next, the Cauchy-Schwarz and Poincaré inequalities lead to the following bound

$$
\begin{aligned}
a\left(u_{b h}, z_{h}\right) \mid & =\left|\varepsilon\left(\nabla u_{b h}, \nabla z_{h}\right)_{\Omega}+\left(\boldsymbol{b} \cdot \nabla u_{b h}, z_{h}\right)_{\Omega}+\left(\sigma u_{b h}, z_{h}\right)\right| \\
& \leq \varepsilon\left|u_{b h}\right|_{1, \Omega}\left|z_{h}\right|_{1, \Omega}+\|\boldsymbol{b}\|_{\infty, \Omega}\left\|u_{b h}\right\|_{1, \Omega}\left\|z_{h}\right\|_{0, \Omega}+C \sigma\left\|u_{b h}\right\|_{0, \Omega}\left\|z_{h}\right\|_{0, \Omega} \\
& \leq C\left\|u_{b h}\right\|_{1, \Omega}\left|z_{h}\right|_{1, \Omega} .
\end{aligned}
$$

In addition, using the shape regularity of the mesh sequence, $\alpha_{E}(\cdot) \leq 1$, and the local trace inequality, we arrive at

$$
\begin{aligned}
\left|d_{h}\left(z_{h}+u_{b h} ; u_{b h}, z_{h}\right)\right| & =\sum_{E \in \mathscr{E}_{h}} \gamma_{0} h_{E}^{d} \alpha_{E}\left(z_{h}+u_{b h}\right)\left(\partial_{t} u_{b h}, \partial_{t} z_{h}\right)_{E} \\
& \leq \sum_{E \in \mathscr{E}_{h}} \gamma_{0} h_{E}^{d}\left\|\partial_{t} u_{b h}\right\|_{0, E}\left\|\partial_{t} z_{h}\right\|_{0, E} \\
& \leq C h\left|u_{b h}\right|_{1, \Omega}\left|z_{h}\right|_{1, \Omega} .
\end{aligned}
$$

We can thus conclude that

$$
\left[T_{h} z_{h}, z_{h}\right] \geq \varepsilon\left|z_{h}\right|_{1, \Omega}^{2}-C\left\|u_{b h}\right\|_{1, \Omega}\left|z_{h}\right|_{1, \Omega}-\|f\|_{0, \Omega}\left\|z_{h}\right\|_{0, \Omega} .
$$

The claimed result arises by applying the Poincaré and Young inequalities to the last relation.

The solvability of the nonlinear problem (2.2) appears as a consequence of the above result and Brower's fixed point theorem.

Theorem 1 The discrete problem (2.2) has at least one solution. Moreover, if $C_{\text {lip }} \gamma_{0} h<\varepsilon$, where $C_{\text {lip }}$ is the constant from Lemma 2, then the solution is unique.

Proof First, since the bilinear form $a(\cdot, \cdot)$ is continuous, and $d_{h}(\cdot ; \cdot, \cdot)$ is Lipschitz continuous, then the operator $T_{h}$ is Lipschitz continuous. Next, in view of (2.18), for any $z_{h} \in \mathcal{V}_{h}^{0}$ such that

$$
\left|z_{h}\right|_{1, \Omega}^{2}=2 \frac{c_{2}\left(\left\|u_{b h}\right\|_{1, \Omega}^{2}+\|f\|_{0, \Omega}^{2}\right)}{c_{1}}
$$

Lemma 3 gives

$$
\left[T_{h} z_{h}, z_{h}\right]=c_{2}\left(\left\|u_{b h}\right\|_{0, \Omega}^{2}+\|f\|_{0, \Omega}^{2}\right)>0 .
$$

Then, using a consequence of Brower's fixed point Theorem (see [11, Corollary 1.1, Ch. IV]), there exists $\tilde{v}_{h} \in \mathcal{V}_{h}^{0}$ such that $T_{h}\left(\tilde{v}_{h}\right)=0$. Hence, $u_{h}:=\tilde{v}_{h}+u_{b h}$ solves (2.2). 
In order to prove uniqueness, let $u_{h}^{1}, u_{h}^{2}$ be two solutions of (2.2). Then, using (2.2) for both solutions, denoting $\tilde{e}_{h}:=u_{h}^{1}-u_{h}^{2}$, and using the Lipschitz continuity of $d_{h}(\cdot ; \cdot, \cdot)$, we obtain

$$
\varepsilon\left|\tilde{e}_{h}\right|_{1, \Omega}^{2} \leq a\left(\tilde{e}_{h}, \tilde{e}_{h}\right)=-d_{h}\left(u_{h}^{1} ; u_{h}^{1}, \tilde{e}_{h}\right)+d_{h}\left(u_{h}^{2} ; u_{h}^{2}, \tilde{e}_{h}\right) \leq C_{\operatorname{lip}} \gamma_{0} h\left|\tilde{e}_{h}\right|_{1, \Omega}^{2}
$$

This leads to

$$
\left(\varepsilon-C_{\operatorname{lip}} \gamma_{0} h\right)\left|\tilde{e}_{h}\right|_{1, \Omega}^{2} \leq 0,
$$

which, using that $\tilde{e}_{h} \in H_{0}^{1}(\Omega)$, finishes the proof.

\subsection{The discrete maximum principle}

This section is devoted to prove that method (2.2) preserves positivity. For this, we will impose the following geometric hypothesis on the mesh. This hypothesis can be tracked back to [22], and in two space dimensions it reduces to impose that the mesh is Delaunay.

Assumption 1 (Hypothesis of Xu and Zikatanov, cf. [22]) For every internal edge $E \in \mathscr{E}_{h}$ with end points $x_{i}$ and $x_{j}$ the following inequality holds

$$
\frac{1}{d(d-1)} \sum_{K \in \omega_{E}}\left|\omega_{i j}^{K}\right| \cot \left(\theta_{i j}^{K}\right) \geq 0
$$

where $\theta_{i j}^{K}$ is the angle between the two facets in $K$ opposite to $x_{i}$ and $x_{j}$ (denoted by $F_{i, K}$ and $F_{j, K}$, respectively), and $\omega_{i j}^{K}$ is the $(d-2)$-dimensional simplex $F_{i, K} \cap F_{j, K}$ opposite to the edge $E$.

We now introduce the discrete analogue of the maximum principle. This definition is related to the one from [7], and it leads to results which are, essentially, identical to those from that reference.

Definition $2(D M P)$ The semilinear form $a_{h}(\cdot ; \cdot)$ is said to satisfy the strong DMP property if the following holds: for all $u_{h} \in \mathcal{V}_{h}$ and for all interior vertices $x_{i}$, if $u_{h}$ is locally minimal (resp. maximal) on the vertex $x_{i}$ over the macro-element $\Omega_{i}$, then there exist negative quantites $\left(c_{E}\right)_{E \in \mathscr{E}_{i}}$ such that

$$
a_{h}\left(u_{h} ; \psi_{i}\right) \leq \sum_{E \in \mathscr{E}_{i}} c_{E}\left|\partial_{t} u_{h}\right|_{E} \mid,
$$

[resp. $\left.a_{h}\left(u_{h} ; \psi_{i}\right) \geq-\sum_{E \in \mathscr{E}_{i}} c_{E}\left|\partial_{t} u_{h}\right|_{E} \mid\right]$. Furthermore, we will say that the semilinear form satisfies the weak DMP property, related to local minima, if (2.28) holds only under the additional assumption that the local minimum above is supposed to be negative. 
A direct consequence of this definition is the following result analoguous to that of [7, Proposition 2.5]. We reproduce the proof here for the reader's convenience.

Lemma 4 Assume that the semilinear form $a_{h}(\cdot ; \cdot)$ satisfies the DMP property. Assume that $u_{h} \in \mathcal{V}_{h}$ solves (2.2) and that $f \geq 0$. Then $u_{h}$ reaches its minimum on the boundary $\partial \Omega$ and for the weak DMP-property, if $g \geq 0$, then $u_{h} \geq 0$ in $\Omega$.

Proof Assume that the DMP is satisfied and $u_{h}$ reaches its minimum in an interior vertex $x_{i}$. Since $a_{h}(\cdot ; \cdot)$ satisfies $(2.28), u_{h}$ is constant over $\Omega_{i}$, implying that the minimum is taken in all vertices $x_{j} \in \Omega_{i}$. Repeating the argument we eventually deduce that the minimum is reached on the boundary.

The following result states the DMP for (2.2).

Theorem 2 Let us suppose that the mesh $\mathscr{T}_{h}$ satisfies Assumption 1, and that the parameter $\gamma_{0}$ is large enough. Then, the semilinear form $a_{h}(\cdot ; \cdot)$ satisfies the weak $D M P$ property for $\sigma>0$ and the strong DMP-property for $\sigma=0$.

Proof Let us suppose that $u_{h}$ has a negative local minimum at an interior node $x_{i}$. Then, $\alpha_{E}\left(u_{h}\right)=1$ for all $E \in \mathscr{E}_{i}$, which gives

$$
\begin{aligned}
a_{h}\left(u_{h} ; \psi_{i}\right)= & \left(\sigma u_{h}, \psi_{i}\right)_{\Omega}+\varepsilon\left(\nabla u_{h}, \nabla \psi_{i}\right)_{\Omega}+\left(\boldsymbol{b} \cdot \nabla u_{h}, \psi_{i}\right)_{\Omega} \\
& +\sum_{E \in \mathscr{E}_{i}} \gamma_{0} h_{E}^{d}\left(\partial_{t} u_{h}, \partial_{t} \psi_{i}\right)_{E} .
\end{aligned}
$$

We will analyse the expression above term-by-term. First, if $u_{h} \leq 0$ in the support of $\psi_{i}$, then $\left(\sigma u_{h}, \psi_{i}\right)_{\Omega} \leq 0$. Let us suppose now that $u_{h}$ changes sign in the support of $\psi_{i}$, and let $K \in \Omega_{i}$ be an element in which $u_{h}$ changes sign. Let $x_{k}$ be a node in $K$ such that $u_{h}\left(x_{k}\right) \geq 0$, and let $E_{i k}$ be the edge connecting these two nodes. Then, using the Cauchy-Schwarz inequality, a Poincaré inequality in $K$, and the shape regularity of the mesh sequence, we arrive at

$$
\begin{aligned}
\left(\sigma u_{h}, \psi_{i}\right)_{K} & \leq \sigma\left\|u_{h}\right\|_{0, K}\left\|\psi_{i}\right\|_{0, K} \\
& \leq C \sigma h_{K}^{\frac{d}{2}}\left\|u_{h}\right\|_{0, K} \\
& \leq C \sigma h_{K}^{d} h_{E_{i k}}\left|\partial_{t} u_{h}\right|_{E_{i k}} \mid
\end{aligned}
$$

Then, adding up over all $K \in \Omega_{i}$ and using the shape regularity of the mesh sequence we obtain

$$
\left(\sigma u_{h}, \psi_{i}\right)_{\Omega} \leq C_{0} \sigma \sum_{E \in \mathcal{E}_{i}} h_{E}^{d+1}\left|\partial_{\mathbf{t}} u_{h}\right|_{E} \mid .
$$

Also, as in [7] (see also [21]), Assumption 1 on the mesh leads to

$$
\varepsilon\left(\nabla u_{h}, \nabla \psi_{i}\right)_{\Omega} \leq 0
$$


Moreover $\sum_{j=1}^{N} \psi_{j}=1$ gives $\sum_{j \in S_{i}}\left(\boldsymbol{b} \cdot \nabla \psi_{j}, \psi_{i}\right)_{\Omega}=0$, and then

$$
\begin{aligned}
\left(\boldsymbol{b} \cdot \nabla u_{h}, \psi_{i}\right)_{\Omega} & =\sum_{j \in S_{i}}\left(\boldsymbol{b} \cdot \nabla \psi_{j}, \psi_{i}\right)_{\Omega} u_{h}\left(x_{j}\right)+\left(\boldsymbol{b} \cdot \nabla \psi_{i}, \psi_{i}\right)_{\Omega} u_{h}\left(x_{i}\right) \\
& =\sum_{j \in S_{i}}\left(\boldsymbol{b} \cdot \nabla \psi_{j}, \psi_{i}\right)_{\Omega}\left(u_{h}\left(x_{j}\right)-u_{h}\left(x_{i}\right)\right) \\
& =\sum_{E \in \mathscr{E}_{i}}\left(\boldsymbol{b} \cdot \nabla \psi_{j}, \psi_{i}\right)_{\Omega} h_{E}\left|\partial_{t} u_{h}\right|_{E} \mid
\end{aligned}
$$

which, using the shape regularity of the mesh sequence gives

$$
\left(\boldsymbol{b} \cdot \nabla u_{h}, \psi_{i}\right)_{\Omega} \leq \sum_{E \in \mathscr{E}_{i}} C_{1}\|\boldsymbol{b}\|_{\infty, E} h_{E}^{d}\left|\partial_{t} u_{h}\right|_{E} \mid .
$$

Finally, since $u_{h}\left(x_{i}\right)$ is a local minimum, then in every $E \in \mathscr{E}_{i}, \partial_{t} u_{h}$ and $\partial_{t} \psi_{i}$ have different signs (independently of the orientation of the tangential vector in $E$ ), which gives

$$
\sum_{E \in \mathscr{E}_{i}} \gamma_{0} h_{E}^{d}\left(\partial_{t} u_{h}, \partial_{t} \psi_{i}\right)_{E}=-\sum_{E \in \mathscr{E}_{i}} \gamma_{0} h_{E}^{d}\left|\partial_{t} u_{h}\right|_{E} \mid .
$$

Hence, gathering all the above computations, we arrive at

$$
a_{h}\left(u_{h} ; \psi_{i}\right) \leq-\sum_{E \in \mathscr{E}_{i}}\left(\gamma_{0}-C_{0} \sigma h_{E}-C_{1}\|\boldsymbol{b}\|_{\infty, E}\right) h_{E}^{d}\left|\partial_{t} u_{h}\right|_{E} \mid,
$$

and the result follows assuming that $\gamma_{0}>C_{0} \sigma h_{E}+C_{1}\|\boldsymbol{b}\|_{\infty, E}$. Finally, we notice that if $\sigma=0$ then the sign of the strict minimum is irrelevant, which proves the strong DMP property.

Remark 2 It is interesting to remark that the hypothesis on the meshes of the triangulation can be avoided if the problem is supposed to be strongly convection-dominated. In fact, following analogous steps to those used to prove (2.32) we can arrive at

$$
\varepsilon\left(\nabla u_{h}, \nabla \psi_{i}\right)_{\Omega}=\varepsilon \sum_{E \in \mathscr{E}_{i}}\left(\nabla \psi_{j}, \nabla \psi_{i}\right)_{\Omega} h_{E}\left|\partial_{t} u_{h}\right| \leq \sum_{E \in \mathscr{E}_{i}} C_{2} \varepsilon h_{E}^{d-1}\left|\partial_{t} u_{h}\right|
$$

Replacing this into the steps leading to (2.35) gives

$$
a_{h}\left(u_{h} ; \psi_{i}\right) \leq-\sum_{E \in \mathscr{E}_{i}}\left(\gamma_{0}-C_{0} \sigma h_{E}-C_{1}\|\boldsymbol{b}\|_{\infty, E}-C_{2} \varepsilon h_{E}^{-1}\right) h_{E}^{d}\left|\partial_{t} u_{h}\right|,
$$

and the proof follows by assuming that $\gamma_{0}>C_{0} \sigma h_{E}+C_{1}\|\boldsymbol{b}\|_{\infty, E}+C_{2} \varepsilon h_{E}^{-1}$.

The last result is only interesting if $\varepsilon h_{E}^{-1}$ stays bounded, which means this is applicable only in the case the problem is highly convection-dominated. In this sense, the method proposed in this work can be applied to scalar conservation laws, regardless of the geometrical impositions on the mesh. Similar results have been obtained recently in $[12,13]$. 


\section{Convergence}

The error will be analysed using the following norm:

$$
\left\|v_{h}\right\|_{h}^{2}:=\sigma\left\|v_{h}\right\|_{0, \Omega}^{2}+\varepsilon\left|v_{h}\right|_{1, \Omega}^{2}+d_{h}\left(u_{h} ; v_{h}, v_{h}\right) .
$$

This norm is not only mesh-dependent, but also depends on the discrete solution. The inclusion of the last term in it is made mostly for convenience, but the fact that it controls the usual $H^{1}(\Omega)$-norm (weighted by physical coefficients) guarantees that the convergence of the method is valid with respect to the standard norm as well. As usual, the error $e:=u-u_{h}$ is split as follows

$$
e=u-u_{h}=\left(u-i_{h} u\right)+\left(i_{h} u-u_{h}\right):=\rho_{h}+e_{h},
$$

where $i_{h}: C^{0}(\bar{\Omega}) \cap H_{0}^{1}(\Omega) \rightarrow \mathcal{V}_{h}^{0}$ stands for the Clément interpolation operator. Using standard interpolation estimates (see [8]), the fact that $\alpha_{E}(\cdot) \leq 1$, and the shape regularity of the mesh sequence, the following bound for $\rho_{h}$ follows:

$$
\left\|\rho_{h}\right\|_{h} \leq C\left(\varepsilon^{\frac{1}{2}}+\sigma^{\frac{1}{2}} h+\gamma_{0} h^{\frac{1}{2}}\right) h\|u\|_{2, \Omega} .
$$

The next result states a bound for $e_{h}$.

Lemma 5 Let us suppose $u \in H^{2}(\Omega) \cap H_{0}^{1}(\Omega)$. Then, there exists $C>0$, independent of $h$ and $\varepsilon$, such that

$$
\left\|e_{h}\right\|_{h} \leq C\left(\varepsilon+\sigma^{-1}\left\{\|\boldsymbol{b}\|_{\infty, \Omega}^{2}+\sigma^{2}\right\}\right)^{\frac{1}{2}} h\|u\|_{2, \Omega}+C h^{\frac{1}{2}}\|u\|_{1, \Omega}
$$

Proof First, from the definition of $a$ and $d_{h}$ we get

$$
\begin{aligned}
\left\|e_{h}\right\|_{h}^{2} & =a\left(e_{h}, e_{h}\right)+d_{h}\left(u_{h} ; e_{h}, e_{h}\right) \\
& =a\left(i_{h} u, e_{h}\right)-\left\{a\left(u_{h}, e_{h}\right)+d_{h}\left(u_{h} ; u_{h}, e_{h}\right)\right\}+d_{h}\left(u_{h} ; i_{h} u, e_{h}\right) \\
& =-a\left(\rho_{h}, e_{h}\right)+d_{h}\left(u_{h} ; i_{h} u, e_{h}\right) .
\end{aligned}
$$

Next, the continuity of $a$ gives

$$
\begin{aligned}
a\left(\rho_{h}, e_{h}\right) & \leq\left(\sigma\left\|\rho_{h}\right\|_{0, \Omega}^{2}+\left[\varepsilon+\sigma^{-1}\|\boldsymbol{b}\|_{\infty, \Omega}^{2}\right]\left|\rho_{h}\right|_{1, \Omega}^{2}\right)^{\frac{1}{2}}\left\|e_{h}\right\|_{h} \\
& \leq C\left(\varepsilon^{\frac{1}{2}}+\sigma^{-1 / 2}\|\boldsymbol{b}\|_{\infty, \Omega}+\sigma^{\frac{1}{2}} h\right) h\|u\|_{2, \Omega}\left\|e_{h}\right\|_{h} .
\end{aligned}
$$

Moreover, since $d_{h}\left(u_{h} ; \cdot, \cdot\right)$ is a symmetric positive semi-definite bilinear form it satisfies Cauchy-Schwarz's inequality, which gives

$$
d_{h}\left(u_{h} ; i_{h} u, e_{h}\right) \leq d_{h}\left(u_{h} ; i_{h} u, i_{h} u\right)^{\frac{1}{2}} d_{h}\left(u_{h} ; e_{h}, e_{h}\right)^{\frac{1}{2}} \leq d_{h}\left(u_{h} ; i_{h} u, i_{h} u\right)^{\frac{1}{2}}\left\|e_{h}\right\|_{h}
$$


Then, inserting (3.6) and (3.7) into (3.5), and using Young's inequality, we arrive at

$$
\left\|e_{h}\right\|_{h}^{2} \leq C\left(\varepsilon^{\frac{1}{2}}+\sigma^{-1 / 2}\|\boldsymbol{b}\|_{\infty, \Omega}+\sigma^{\frac{1}{2}} h\right)^{2} h^{2}\|u\|_{2, \Omega}^{2}+C d_{h}\left(u_{h} ; i_{h} u, i_{h} u\right) .
$$

It only remains to bound the consistency error $d_{h}\left(u_{h} ; i_{h} u, i_{h} u\right)$ in (3.8). The definition of $d_{h}(\cdot ; \cdot, \cdot), \alpha_{E}\left(u_{h}\right) \leq 1$, a local trace inequality, the shape regularity of the mesh sequence, and the $H^{1}(\Omega)$-stability of $i_{h}$, give

$$
\begin{aligned}
d_{h}\left(u_{h} ; i_{h} u, i_{h} u\right) & =\sum_{E \in \mathscr{E}_{h}} \gamma_{0} h_{E}^{d} \alpha_{E}\left(u_{h}\right)\left\|\partial_{t} i_{h} u\right\|_{0, E}^{2} \\
& \leq \gamma_{0} h \sum_{E \in \mathscr{E}_{h}} h_{E}^{d-1}\left\|\partial_{t} i_{h} u\right\|_{0, E}^{2} \leq C h\|u\|_{1, \Omega}^{2} .
\end{aligned}
$$

Then, the result arises inserting (3.9) into (3.8).

Collecting (3.3) and Lemma 5 we then obtain the following error estimate for (2.2). Theorem 3 Let us suppose $u \in H^{2}(\Omega) \cap H_{0}^{1}(\Omega)$. Then, there exists $C>0$, independent of $h$ and $\varepsilon$, such that

$$
\|e\|_{h} \leq C\left(\varepsilon+\sigma^{-1}\left\{\|\boldsymbol{b}\|_{\infty, \Omega}^{2}+\sigma^{2}\right\}\right)^{\frac{1}{2}} h\|u\|_{2, \Omega}+C h^{\frac{1}{2}}\|u\|_{1, \Omega} .
$$

The following result states that for meshes which are symmetric with respect to their interior nodes, the method converges with a higher order. This result's main interest lies in the diffusion dominated regime, due to the factor $\varepsilon^{-\frac{1}{2}}$ present in the estimate. The combination of Lipschitz continuity and linearity preservation seems to be novel, and that is why we do detail it now.

Theorem 4 Let us suppose $u \in H^{2}(\Omega) \cap H_{0}^{1}(\Omega)$ and that the mesh is symmetric with respect to its internal nodes. Then, there exists $C>0$, independent of $h$ and $\varepsilon$, such that

$$
\|e\|_{h} \leq C\left(\varepsilon+\sigma^{-1}\left\{\|\boldsymbol{b}\|_{\infty, \Omega}^{2}+\sigma^{2}\right\}\right)^{\frac{1}{2}} h\|u\|_{2, \Omega}+C \frac{h}{\sqrt{\varepsilon}}\|u\|_{1, \Omega} .
$$

Proof It is enough to bound the consistency error $d\left(u_{h} ; i_{h} u, i_{h} u\right)$. We have

$$
\begin{aligned}
d_{h}\left(u_{h} ; i_{h} u, i_{h} u\right) & =\left\{d_{h}\left(u_{h} ; i_{h} u, i_{h} u\right)-d_{h}\left(i_{h} u ; i_{h} u, i_{h} u\right)\right\}+d_{h}\left(i_{h} u ; i_{h} u, i_{h} u\right) \\
& =: \mathrm{I}+\mathrm{II} .
\end{aligned}
$$

The first term is bounded as in the proof of Lemma 2. In fact, in that proof, the bound for the second term in (2.10) leads to the following

$$
\begin{aligned}
\mathrm{I} & =\sum_{E \in \mathscr{E}_{h}}\left(\alpha_{E}\left(u_{h}\right)-\alpha_{E}\left(i_{h} u\right)\right) \gamma_{0} h_{E}^{d}\left(\partial_{t} i_{h} u, \partial_{t} i_{h} u\right)_{E} \\
& \leq C h\left|u_{h}-i_{h} u\right|_{1, \Omega}\left|i_{h} u\right|_{1, \Omega} \\
& \leq \frac{\varepsilon}{2}\left|u_{h}-i_{h} u\right|_{1, \Omega}^{2}+C \frac{h^{2}}{\varepsilon}\|u\|_{1, \Omega}^{2}
\end{aligned}
$$


where we have also used the $H^{1}(\Omega)$-stability of $i_{h}$. To bound II we use the linearity preservation and the Lipschitz continuity of $d_{h}(\cdot ; \cdot, \cdot)$. More precisely, for a given $E \in \mathscr{E}_{h}$ we introduce the function $i_{E} u \in \mathbb{P}_{1}\left(\omega_{E}\right)$ as the unique solution of the problem

$$
\begin{aligned}
\left(\nabla i_{E} u, \nabla \psi\right)_{\omega_{E}} & =(\nabla u, \nabla \psi)_{\omega_{E}} \quad \forall \psi \in \mathbb{P}_{1}\left(\omega_{E}\right) \\
\left(i_{E} u, 1\right)_{\omega_{E}} & =(u, 1)_{\omega_{E}} .
\end{aligned}
$$

Using standard finite element approximation results (see [8]), $i_{E} u$ satisfies

$$
\left|u-i_{E} u\right|_{1, \omega_{E}} \leq C h_{E}|u|_{2, \omega_{E}}
$$

Since the mesh is symmetric with respect to its internal nodes, $\alpha_{E}\left(i_{E} u\right)=0$. Then, proceeding as in the bound for I we obtain

$$
\begin{aligned}
\mathrm{II} & =\sum_{E \in \mathscr{E}_{h}}\left(\alpha_{E}\left(i_{h} u\right)-\alpha_{E}\left(i_{E} u\right)\right) \gamma_{0} h_{E}^{d}\left(\partial_{\boldsymbol{t}} i_{h} u, \partial_{t} i_{h} u\right)_{E} \\
& \leq C h\left\{\sum_{E \in \mathscr{E}_{h}}\left|i_{h} u-i_{E} u\right|_{1, \omega_{E}}^{2}\right\}^{\frac{1}{2}}\left|i_{h} u\right|_{1, \Omega} \\
& \leq C h^{2}|u|_{2, \Omega}\|u\|_{1, \Omega} .
\end{aligned}
$$

Then, inserting (3.13) and (3.16) into (3.12) we obtain

$$
d_{h}\left(u_{h} ; i_{h} u, i_{h} u\right) \leq \frac{\varepsilon}{2}\left|u_{h}-i_{h} u\right|_{1, \Omega}^{2}+C \frac{h^{2}}{\varepsilon}\|u\|_{1, \Omega}^{2}+C h^{2}|u|_{2, \Omega}\|u\|_{1, \Omega}
$$

and the result follows by rearranging terms.

\section{A link to algebraic flux correction schemes}

Method (2.2) has been presented having as motivation the study of the effect of adding edge-based diffusion into the equations to impose the discrete maximum principle. Another family of methods that are built with the same purpose is the AFC schemes. This section is devoted to study the relationship between the two approaches, and that is why we now summarise the main building principles of AFC schemes.

The starting point of an algebraic flux-correction scheme is a discretisation of the convection-diffusion-reaction equation which leads to the linear system

$$
\mathbb{A} U=\mathbb{G},
$$


where $\mathbb{A}=\left(a_{i j}\right)_{i, j=1}^{N}, \mathrm{U}=\left\{u_{h}\left(x_{i}\right)\right\}_{i=1}^{N}$ and $\mathbb{G}=\left\{g_{i}\right\}_{i=1}^{N}$. The first step of these schemes is to identify which parts of the system matrix $\mathbb{A}$ are responsible for the violation of the discrete maximum principle. To achieve this, the diffusion matrix $\mathbb{D}=\left(d_{i j}\right)_{i, j=1}^{N}$ is built, where

$$
d_{i j}=d_{j i}=-\max \left\{a_{i j}, 0, a_{j i}\right\} \quad \forall i \neq j \quad d_{i i}=-\sum_{j \neq i} d_{i j} .
$$

Adding $\mathbb{D U}$ both sides of (4.1) we obtain

$$
\tilde{\mathbb{A}} \mathrm{U}=\mathbb{G}+\mathbb{D} \mathrm{U}
$$

where $\tilde{\mathbb{A}}:=\mathbb{A}+\mathbb{D}$. Since the matrix $\tilde{\mathbb{A}}$ fullfils the hypothesis to guarantee the discrete maximum principle, then the oscillations that appear in a non-stabilised discretisation (4.1) are due to the right-hand side. This is why the right-hand side is now rewritten. Using that the row-sums of $\mathbb{D}$ are zero, then

$$
(\mathbb{D} U)_{i}=\sum_{j \neq i} f_{i j} \quad \text { where } f_{i j}=d_{i j}\left(u_{h}\left(x_{j}\right)-u_{h}\left(x_{i}\right)\right)
$$

The quantities $f_{i j}$ are called fluxes. Then, the AFC schemes are based on introducing limiters $\alpha_{i j}\left(u_{h}\right)$ such that $\alpha_{i j} \in[0,1], \alpha_{i j}=\alpha_{j i}$, and $\alpha_{i j}=1$ if $x_{i}$ and $x_{j}$ are both Dirichlet nodes. Then, after introducing these limiters, the method reads as follows:

$$
\mathbb{A} \mathrm{U}_{i}+\sum_{i, j=1}^{N}\left(1-\alpha_{i j}\left(u_{h}\right)\right) d_{i j}\left(u_{h}\left(x_{j}\right)-u_{h}\left(x_{i}\right)\right)=g_{i}
$$

The most popular limiters in practice are Zalesak's limiters (see, Refs. [15-17,23], and the recent review [18] for examples). The analysis of these methods for a class of limiters that includes the Zalesak one has been carried out recently in [2,3]. In particular, in [2] an $O\left(h^{\frac{1}{2}}\right)$ convergence rate was proved for the case in which the mesh used satisfies Assumption 1. In the case of meshes that do not satisfy this assumption, then no convergence can be proved, unless some appropriate modifications are done to the algorithm. This result is optimal, as the numerical results in [2] show.

Following [2], Eq. (4.3) can be written as the following weak problem: find $u_{h} \in \mathcal{V}_{h}$ such that $u_{h}-u_{b h} \in \mathcal{V}_{h}^{0}$, and

$$
a\left(u_{h}, v_{h}\right)+\tilde{d}_{h}\left(u_{h} ; u_{h}, v_{h}\right)=\left(f, v_{h}\right)_{\Omega} \quad \forall v_{h} \in \mathcal{V}_{h}^{0}
$$

where the nonlinear form $\tilde{d}_{h}(\cdot ; \cdot, \cdot)$ is given by

$$
\tilde{d}_{h}\left(u_{h} ; u_{h}, v_{h}\right)=\sum_{i, j=1}^{N}\left(1-\alpha_{i j}\left(u_{h}\right)\right) d_{i j}\left(u_{h}\left(x_{j}\right)-u_{h}\left(x_{i}\right)\right) v_{h}\left(x_{i}\right)
$$


Next, to link this to the method analysed in the last sections, we use the symmetry of $\mathbb{D}$, and of the limiters $\alpha_{i j}=\alpha_{j i}$, and a simple calculation gives:

$$
\begin{aligned}
\tilde{d}_{h}\left(u_{h} ; u_{h}, v_{h}\right)= & \sum_{i>j}\left(1-\alpha_{i j}\left(u_{h}\right)\right) d_{i j}\left(u_{h}\left(x_{j}\right)-u_{h}\left(x_{i}\right)\right) v_{h}\left(x_{i}\right) \\
& +\sum_{i<j}\left(1-\alpha_{i j}\left(u_{h}\right)\right) d_{i j}\left(u_{h}\left(x_{j}\right)-u_{h}\left(x_{i}\right)\right) v_{h}\left(x_{i}\right) \\
= & \sum_{i>j}\left(1-\alpha_{i j}\left(u_{h}\right)\right) d_{i j}\left(u_{h}\left(x_{j}\right)-u_{h}\left(x_{i}\right)\right) v_{h}\left(x_{i}\right) \\
& +\sum_{i>j}\left(1-\alpha_{j i}\left(u_{h}\right)\right) d_{j i}\left(u_{h}\left(x_{i}\right)-u_{h}\left(x_{j}\right)\right) v_{h}\left(x_{j}\right) \\
= & \sum_{i>j}\left(1-\alpha_{i j}\left(u_{h}\right)\right) d_{i j}\left(u_{h}\left(x_{j}\right)-u_{h}\left(x_{i}\right)\right)\left(v_{h}\left(x_{i}\right)-v_{h}\left(x_{j}\right)\right) .
\end{aligned}
$$

Then, since $d_{i j}=0$ for $j \notin S_{i}, \tilde{d}_{h}(\cdot ; \cdot, \cdot)$ can be rewritten as

$$
\tilde{d}_{h}\left(u_{h} ; u_{h}, v_{h}\right)=\sum_{E \in \mathscr{E}_{h}}\left(1-\alpha_{i j}\left(u_{h}\right)\right)\left|d_{i j}\right| h_{E}\left(\partial_{t} u_{h}, \partial_{t} v_{h}\right)_{E}
$$

where we have adopted the convention that an edge $E \in \mathscr{E}_{h}$ has endpoints $x_{i}$ and $x_{j}$, and used that $\alpha_{i j}=1$ for edges included in the Dirichlet boundary.

Method (2.2) then appears as an algebraic flux-correction scheme, with a different definition of the limiters. Indeed comparing (2.2) with (4.7) we get the equivalent AFC scheme if we choose $\alpha_{i j}\left(u_{h}\right)$ such that

$$
\left(1-\alpha_{i j}\left(u_{h}\right)\right)\left|d_{i j}\right| h_{E}=\gamma_{0} h_{E}^{d} \alpha_{E}\left(u_{h}\right)
$$

The new definition of the limiters made it possible to write some convergence and existence results, also present in [2], in a more precise way, and improve in some of them. In particular, the new limiters make it possible to prove convergence for general meshes, as well as to prove uniqueness of solutions and optimal convergence in the diffusion dominated regime.

\section{Numerical results}

In this section we present three sets of numerical results for bi-dimensional problems. All three cases are set in $\Omega=(0,1)^{2}$. The nonlinear system (2.2) has been solved using the following fixed-point algorithm with damping: starting with the Galerkin solution $u_{h}^{0}$, then compute a sequence $\left\{u_{h}^{k}\right\}$ defined by

$$
u_{h}^{k+1}=u_{h}^{k}+\omega\left(\tilde{u}_{h}^{k+1}-u_{h}^{k}\right) \quad k=0,1,2, \ldots
$$


where $\omega \in(0,1)$ is a damping parameter, and $\tilde{u}_{h}^{k+1}$ solves: $\tilde{u}_{h}^{k+1}-u_{b h} \in \mathcal{V}_{h}^{0}$, and

$$
a\left(\tilde{u}_{h}^{k+1}, v_{h}\right)+d_{h}\left(u_{h}^{k} ; \tilde{u}_{h}^{k+1}, v_{h}\right)=\left(f, v_{h}\right) \quad \forall v_{h} \in \mathcal{V}_{h}^{0}
$$

In all our calculations we have used $\omega=0.1$, and stopped the iterations when the residual $\boldsymbol{R}^{k}:=\left(a_{h}\left(u_{h}^{k+1} ; \psi_{i}\right)-\left(f, \psi_{i}\right)_{\Omega}\right)_{i=1, \ldots, \operatorname{dim}\left(\mathcal{V}_{h}^{0}\right)}$ has an euclidean norm smaller than, or equal to, $10^{-8}$.

\subsection{Convergence for a smooth solution}

We take $\boldsymbol{b}=(2,1), \sigma=1$, and different values for $\varepsilon$. We have selected the right-handside and boundary conditions in such a way that the solution is given by $u(x, y)=$ $\sin (2 \pi x) \sin (2 \pi y)$. The meshes used were the three-directional mesh (c) and the non-Delaunay mesh (d) in Fig. 1. In these calculations we have used $\gamma_{0}=3$ and $p=4$.

The results in Tables 1, 2, 3 and 4 match the theoretical results. In particular we observe a first order convergence in the diffusion-dominated regime for the mesh (c), as predicted by Theorem 4, and a second order convergence in the $L^{2}$ norm of the error for both the convection and diffusion-dominated regimes. The latter is in accordance with the empirical observations that linearity preservation implies such a convergence. For mesh (d), which is non-symmetric, and hence the method is no longer linearity preserving, we can observe a first order convergence in both regimes. This convergence is not affected by the non-Delaunay character of the mesh.

We finish this example by a deeper study of the behavior of the nonlinear fixed-point iteration with respect to the value of $p$. The results are reported in Table 5. For these results, we have used the three-directional mesh (c), with $l=5$. We can observe that, for the values of $p$ ranging from 1 to 10 the iterations needed to reach convergence are essentially independent of the value of $p$. This behavior is kept until a value around 20, and then some non-convergence is observed in the scheme. Here, by non-convergence we mean that the desired residual reduction has not been achieved after 5000 iterations. The same qualitative behavior has been observed for other meshes, and the two other settings presented later. In those cases, non-convergence has been observed starting at

Table $1 \varepsilon=10^{-6}$, numerical results for grid (c)

\begin{tabular}{lllllll}
\hline$l$ & $\left\|u-u_{h}\right\|_{0, \Omega}$ & Ord. & $\left|u-u_{h}\right|_{1, \Omega}$ & Ord. & $\left\|u-u_{h}\right\|_{h}$ & Ord. \\
\hline 3 & 0.49391 & - & 4.38896 & - & 3.62380 & - \\
4 & 0.47965 & 0.04 & 4.26871 & 0.04 & 3.08479 & 0.23 \\
5 & 0.19110 & 1.33 & 2.71665 & 0.65 & 1.08371 & 1.51 \\
6 & 0.04080 & 2.23 & 1.55469 & 0.81 & 0.22671 & 2.26 \\
7 & 0.00683 & 2.58 & 0.64692 & 1.27 & 0.03904 & 2.54 \\
8 & 0.00119 & 2.52 & 0.27480 & 1.24 & 0.00689 & 2.50 \\
\hline
\end{tabular}


Table $2 \varepsilon=1$, numerical results for grid (c)

\begin{tabular}{lllllll}
\hline$l$ & $\left\|u-u_{h}\right\|_{0, \Omega}$ & Ord. & $\left|u-u_{h}\right|_{1, \Omega}$ & Ord. & $\left\|u-u_{h}\right\|_{h}$ & Ord. \\
\hline 3 & 0.38594 & - & 3.48242 & - & 5.44504 & - \\
4 & 0.16557 & 1.22 & 1.90920 & 0.87 & 2.26966 & 1.26 \\
5 & 0.03268 & 2.34 & 0.89029 & 1.10 & 0.92785 & 1.29 \\
6 & 0.00612 & 2.42 & 0.43637 & 1.03 & 0.43912 & 1.08 \\
7 & 0.00141 & 2.12 & 0.21800 & 1.00 & 0.21818 & 1.01 \\
8 & 0.00035 & 2.02 & 0.10903 & 1.00 & 0.10904 & 1.00 \\
\hline
\end{tabular}

Table $3 \varepsilon=10^{-6}$, numerical results for grid (d)

\begin{tabular}{lllllll}
\hline$l$ & $\left\|u-u_{h}\right\|_{0, \Omega}$ & Ord. & $\left|u-u_{h}\right|_{1, \Omega}$ & Ord. & $\left\|u-u_{h}\right\|_{h}$ & Ord. \\
\hline 3 & 0.48754 & - & 4.33607 & - & 5.06989 & - \\
4 & 0.45680 & 0.09 & 4.11426 & 0.08 & 2.93242 & 0.79 \\
5 & 0.17080 & 1.42 & 3.15455 & 0.38 & 1.05213 & 1.48 \\
6 & 0.04330 & 1.98 & 2.23948 & 0.49 & 0.26065 & 2.01 \\
7 & 0.01165 & 1.89 & 1.72410 & 0.38 & 0.05482 & 2.25 \\
8 & 0.00474 & 1.30 & 1.63424 & 0.08 & 0.02087 & 1.39 \\
\hline
\end{tabular}

Table $4 \varepsilon=1$, numerical results for grid (d)

\begin{tabular}{lllllll}
\hline$l$ & $\left\|u-u_{h}\right\|_{0, \Omega}$ & Ord. & $\left|u-u_{h}\right|_{1, \Omega}$ & Ord. & $\left\|u-u_{h}\right\|_{h}$ & Ord. \\
\hline 3 & 0.38351 & - & 3.52996 & - & 5.57464 & - \\
4 & 0.16616 & 1.21 & 2.00539 & 0.82 & 2.41681 & 1.21 \\
5 & 0.04513 & 1.88 & 0.98086 & 1.03 & 1.03172 & 1.23 \\
6 & 0.01277 & 1.82 & 0.48118 & 1.03 & 0.48720 & 1.08 \\
7 & 0.00423 & 1.59 & 0.23973 & 1.01 & 0.24059 & 1.02 \\
8 & 0.00163 & 1.38 & 0.11982 & 1.00 & 0.11998 & 1.00 \\
\hline
\end{tabular}

Table 5 Iterations needed to reach convergence

\begin{tabular}{|c|c|c|c|c|c|c|c|c|c|c|c|c|}
\hline$p$ & 1 & 2 & 3 & 4 & 5 & 6 & 7 & 8 & 9 & 10 & 15 & 20 \\
\hline Iter. & 224 & 218 & 261 & 262 & 278 & 286 & 211 & 227 & 197 & 197 & 218 & 206 \\
\hline
\end{tabular}

values of about 10 or 15 , depending on the case. Then, we believe that it is safe to use this scheme for values of $p$ not much higher than 10. Of course, further work could be used to find the right damping parameters for each case, but this would come at the price of having to perform much more iterations. 


\subsection{A problem with one inner layer, and a rotating convective field}

We use $\varepsilon=10^{-5}, f=0, \sigma=0, \boldsymbol{b}=(-y, x)$, homogeneous Neumann boundary conditions on exit, and

$$
g(x, y)= \begin{cases}1 & \text { if } x \leq 0.5 \\ 0 & \text { else }\end{cases}
$$

as Dirichlet condition at entry. We have solved this problem on a uniform refinement of the three-directional from mesh (c) in Fig. 1. The parameter $\gamma_{0}$ has been set to 1 , and the results show no violation of the DMP. The results for this case are depicted in Fig. 2. We can observe that the increase in the value of $p$ provides a solution whose inner layer is much sharper than the choice $p=1$. For both higher values for $p$, a similar behaviour to the one in Table 5 was observed in terms of number of iterations needed for convergence.

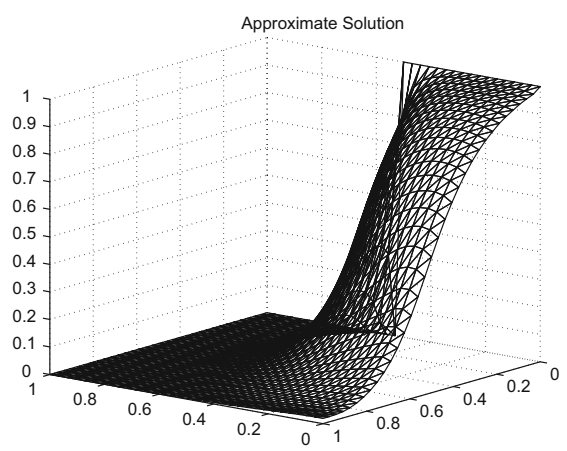

(a)

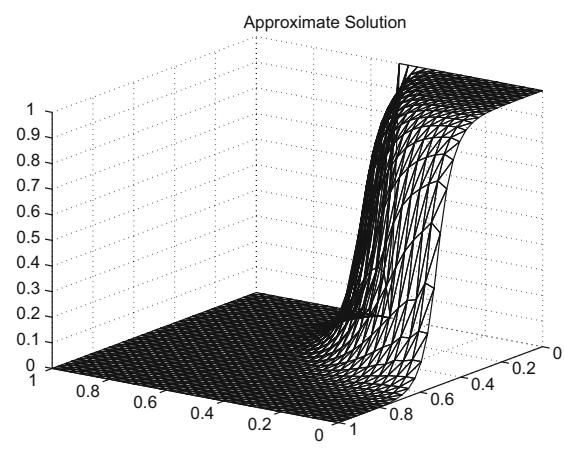

(b)

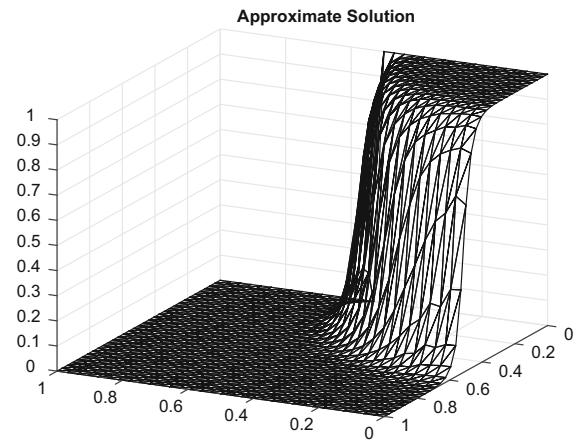

(c)

Fig. 2 Discrete solution for $p=1$ (top left) and $p=4$ (top right), and $p=8$ (bottom) 


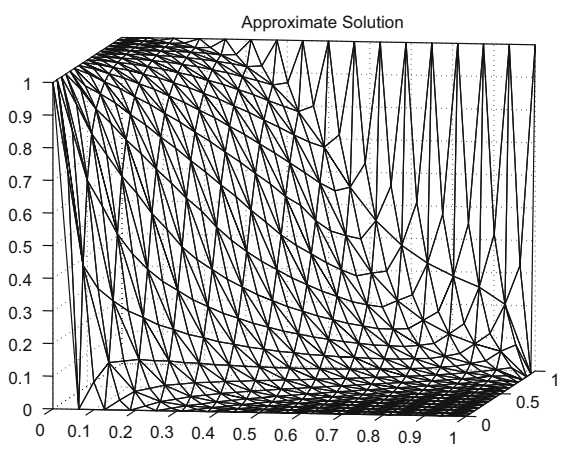

(a)

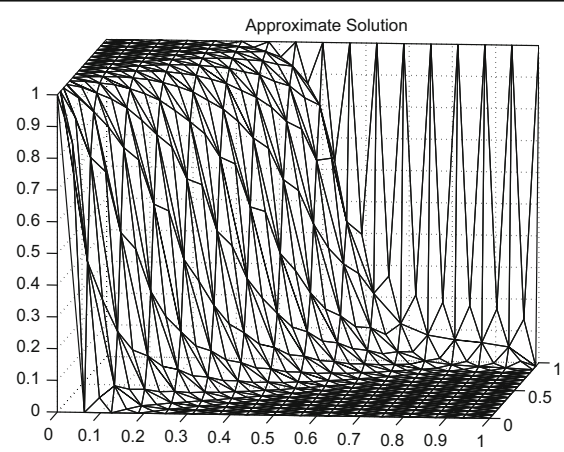

(b)

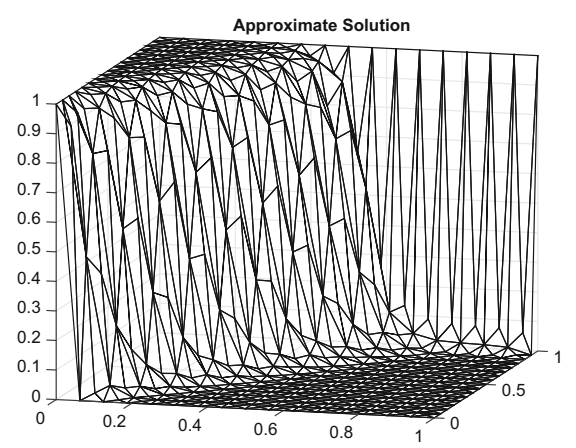

(c)

Fig. 3 Discrete solution for $p=1$ (top left) and $p=4$ (top right), and $p=10$ (bottom)

\subsection{Advection skew to the mesh}

We use $\varepsilon=10^{-5}, f=0, \sigma=0 \boldsymbol{b}=\left(\cos \left(\frac{\pi}{3}\right), \sin \left(\frac{\pi}{3}\right)\right)$, and

$$
g(x, y)= \begin{cases}1 & \text { if } x=0 \text { or } y=1 \\ 0 & \text { else }\end{cases}
$$

as Dirichlet condition. We have solved this problem on a criss-cross mesh as shown in mesh (a) in Fig. 1. We have used the parameter $\gamma_{0}=0.75$, and, again, no violations of the DMP have been observed. The results are depicted in Fig. 3, where we can observe much sharper layers (especially the internal one) when higher values for $p$ have been used. Again, for both higher values for $p$, a similar behaviour to the one in Table 5 was observed in terms of number of iterations needed for convergence.

Acknowledgements The work of GRB and FK has been partially funded by the Leverhulme Trust via the Research Project Grant No. RPG-2012-483. The authors would like to thank Volker John and Petr Knobloch for very helpful discussions.

Open Access This article is distributed under the terms of the Creative Commons Attribution 4.0 International License (http://creativecommons.org/licenses/by/4.0/), which permits unrestricted use, distribution, 
and reproduction in any medium, provided you give appropriate credit to the original author(s) and the source, provide a link to the Creative Commons license, and indicate if changes were made.

\section{References}

1. Badia, S., Hierro, A.: On monotonicity-preserving stabilized finite element approximations of transport problems. SIAM J. Sci. Comput. 36(6), A2673-A2697 (2014). doi:10.1137/130927206

2. Barrenechea, G.R., John, V., Knobloch, P.: Analysis of algebraic flux correction schemes. SIAM J. Numer. Anal. (2015, in press)

3. Barrenechea, G.R., John, V., Knobloch, P.: Some analytical results for an algebraic flux correction scheme for a steady convection-diffusion equation in one dimension. IMA J. Numer. Anal. 35(4), 1729-1756 (2015). doi:10.1093/imanum/dru041

4. Burman, E.: On nonlinear artificial viscosity, discrete maximum principle and hyperbolic conservation laws. BIT 47(4), 715-733 (2007). doi:10.1007/s10543-007-0147-7

5. Burman, E., Ern, A.: Nonlinear diffusion and discrete maximum principle for stabilized Galerkin approximations of the convection-diffusion-reaction equation. Comput. Methods Appl. Mech. Eng. 191(35), 3833-3855 (2002). doi:10.1016/S0045-7825(02)00318-3

6. Burman, E., Ern, A.: Discrete maximum principle for Galerkin approximations of the Laplace operator on arbitrary meshes. C. R. Math. Acad. Sci. Paris 338(8), 641-646 (2004). doi:10.1016/j.crma.2004. 02.010

7. Burman, E., Ern, A.: Stabilized Galerkin approximation of convection-diffusion-reaction equations: discrete maximum principle and convergence. Math. Comput. 74, 1637-1652 (2005)

8. Ern, A., Guermond, J.L.: Theory and Practice of Finite Elements. Springer, New York (2004)

9. Ern, A., Guermond, J.L.: Weighting the edge stabilization. SIAM J. Numer. Anal. 51(3), 1655-1677 (2013). doi:10.1137/120867482

10. Gilbarg, D., Trudinger, N.S.: Elliptic partial differential equations of second order. In: Grundlehren der Mathematischen Wissenschaften [Fundamental Principles of Mathematical Sciences], vol. 224, 2nd edn. Springer, Berlin (1983). doi:10.1007/978-3-642-61798-0

11. Girault, V., Raviart, P.A.: Finite element methods for Navier-Stokes equations. In: Springer Series in Computational Mathematics, vol. 5. Springer, Berlin (1986). doi:10.1007/978-3-642-61623-5

12. Guermond, J.L., Nazarov, M.: A maximum-principle preserving $C^{0}$ finite element method for scalar conservation equations. Comput. Methods Appl. Mech. Eng. 272, 198-213 (2014). doi:10.1016/j.cma. 2013.12.015

13. Guermond, J.L., Nazarov, M., Popov, B., Yang, Y.: A second-order maximum principle preserving Lagrange finite element technique for nonlinear scalar conservation equations. SIAM J. Numer. Anal. 52(4), 2163-2182 (2014). doi:10.1137/130950240

14. John, V., Knobloch, P.: On spurious oscillations at layers diminishing (SOLD) methods for convectiondiffusion equations: part I-a review. Comput. Methods Appl. Mech. Eng. 196, 2197-2215 (2007)

15. Kuzmin, D.: On the design of general-purpose flux limiters for finite element schemes. I. Scalar convection. J. Comput. Phys. 219, 513-531 (2006)

16. Kuzmin, D.: Algebraic flux correction for finite element discretizations of coupled systems. In: Papadrakakis, M., Oñate, E., Schrefler, B. (eds.) Proceedings of the Int. Conf. on Computational Methods for Coupled Problems in Science and Engineering, pp. 1-5. CIMNE, Barcelona (2007)

17. Kuzmin, D.: Linearity-preserving flux correction and convergence acceleration for constrained Galerkin schemes. J. Comput. Appl. Math. 236, 2317-2337 (2012)

18. Kuzmin, D., Hämäläinen, J.: Finite element methods for computational fluid dynamics. In: Computational Science and Engineering, vol. 14. Society for Industrial and Applied Mathematics (SIAM), Philadelphia (2015). (A practical guide)

19. Kuzmin, D., Turek, S.: Flux correction tools for finite elements. J. Comput. Phys. 175(2), 525-558 (2002). doi:10.1006/jcph.2001.6955

20. Löhner, R., Morgan, K., Vahdati, M., Boris, J.P., Book, D.L.: FEM-FCT: combining unstructured grids with high resolution. Commun. Appl. Numer. Methods 4(6), 717-729 (1988). doi:10.1002/cnm. 1630040605

21. Roos, H.G., Stynes, M., Tobiska, L.: Robust Numerical Methods for Singularly Perturbed Differential Equations. Convection-Diffusion-Reaction and Flow Problems, 2nd edn. Springer, Berlin (2008) 
22. Xu, J., Zikatanov, L.: A monotone finite element scheme for convection-diffusion equations. Math. Comput. 68(228), 1429-1446 (1999). doi:10.1090/S0025-5718-99-01148-5

23. Zalesak, S.T.: Fully multidimensional flux-corrected transport algorithms for fluids. J. Comput. Phys. 31, 335-362 (1979) 